\title{
Spectral Behavior of a Low-Cost All-Fiber Component Based on Untapered Multifiber Unions
}

\author{
PALOMA R. HORCHE, MANUEL LÓPEZ-AMO, MIGUEL A. MURIEL, \\ JOSÉ A. MARTIN-PEREDA
}

\begin{abstract}
In this work, untapered maultifiber unions are reported to show a spectral behavior similar to the tapered ones. Their oscillatory behavior does not depend on the biconical regions; hence, a new way to make low-cost all-fiber devices with applications as passive components such optical filters and wavelength mux/demuxs is opened. Two types of multimode fibers have been studied and information about the index proffle influence has been obtained. Polarization insensitivity and temperature stability have been observed.
\end{abstract}

\section{INTRODUCTION}

QEVERAL papers have been published in the last years concerning optical fibers devices. Most applications were related to WDM, sensors, and optical filters. A particular case has received special interest because of its wide variety of applications [1]-[3]. It concerns the use of tapered singlemode fibers and comes from the fact that they are the basis of fused couplers. As it is known, biconically tapered structures are composed of a multimode region located between two single-mode regions with conical transition zones. A structure where these regions are nonexistent is going to be presented in this letter. This passive device can be made just with a multimode fiber spliced between two single-mode fibers. A further difference from previous structures is the number of excited modes in the central region. At tapered fibers, this region is considered as a two-mode waveguide. Our present configuration gives a multimode region where the two-mode approximation is not strictly correct. This structure has been called untapered multifiber unions [4]. Large oscillations in the power transmission, obtained with abruptly tapered slopes, have been shown to be related to the interference process [1] between the excited modes of the multimode zone. In this letter, untapered multifiber unions will be shown to be similar to abruptly tapered fibers, having similar oscillatory behavior and the same filtering applications.

\section{EXPERIMENTAL}

The experimental setup, employed to measure wavelength transmission, is composed of a white light source and spectrum-analyzer system which uses a $\mathrm{Ge}$ detector and a
Michelson interferometer. The FFT of the Michelson interferometer output signal is obtained and displayed. The system has the possibility of obtaining the interferogram and coherence function of the input signal. Due to this fact, information about the output signal modes can be obtained. A cladding modestripper ensures that only the transmitted fundamental-mode power $\left(\mathrm{HE}_{11}\right)$ reaches the detector. It is interesting to point out that the cladding mode-stripper has the same effect whether placed before or after a multimode region.

Our experimental configuration is shown in Fig. 1. Two different types of multimode fibers have been employed. Namely, their difference concerns their refractive index profile, being both shown in Fig. 2, where they are named $F 1$ and F2. It is important to point out the central refractive index dip in the core. With these untapered multifiber unions, some transmitted power measurements have been performed. Different lengths for the multimode zone $L$ have been taken.

Fig. 3 shows the transmitted power versus wavelength for both different multimode fibers (line (a) for $F 1$ and line (b) for $F 2$ ) with $L=12 \mathrm{~cm}$. Line (c) shows the overall system response (light source, monomode fiber, and cladding modestripper).

The peak separation $\Delta \lambda_{p}$ defined as $\Delta \lambda_{p}=\lambda_{p}-\lambda_{p-1}$, where $\lambda_{p}$ is the center wavelength of the $p$ th peak, depends on the working wavelength and is inversely proportional to $L$. For a given $L$, the peak separation increases with the wavelength in the case of $F 1$, and is slightly dependent on the working wavelength for $\boldsymbol{F} 2$. Different peak separations $\Delta \lambda_{p}$, for different multimode fiber lengths $L$, are indicated in Fig. 5.

As it can be seen in Fig. 3, the power oscillation has a certain modulation indicating the existence of more than two modes. Numerical calculations have indicated that three are the modes carrying most of the energy. Because every pair of modes has a different propagation constant difference, different oscillation amplitudes are obtained.

A further experimentally observed effect is the strong influence of the fiber alignment on the spectral behavior. A slight misalignment can induce unwanted modes at the multimode region, altering power behavior.

\section{Comments}

Obtained results with both types of multimode fibers show similar oscillations as previously reported in [5] for tapered 


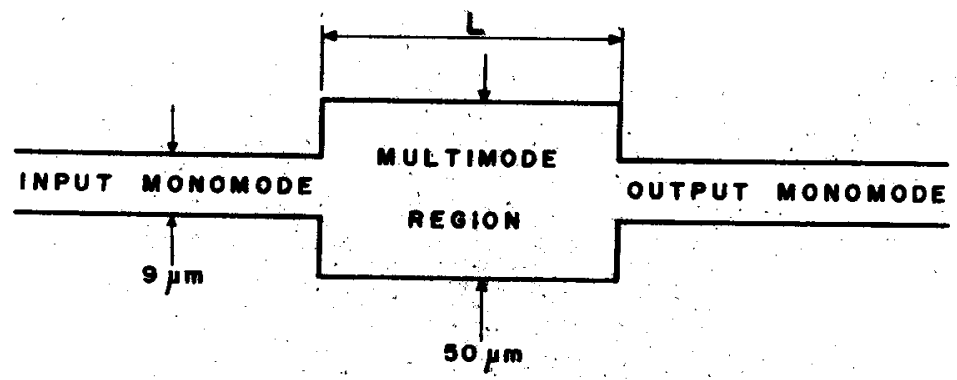

Fig. 1. Structure of untapered multifiber unions. This structure is composed of a multimode region between twó single-mode régions, with no transition zones. $L$ is the multimode region length.

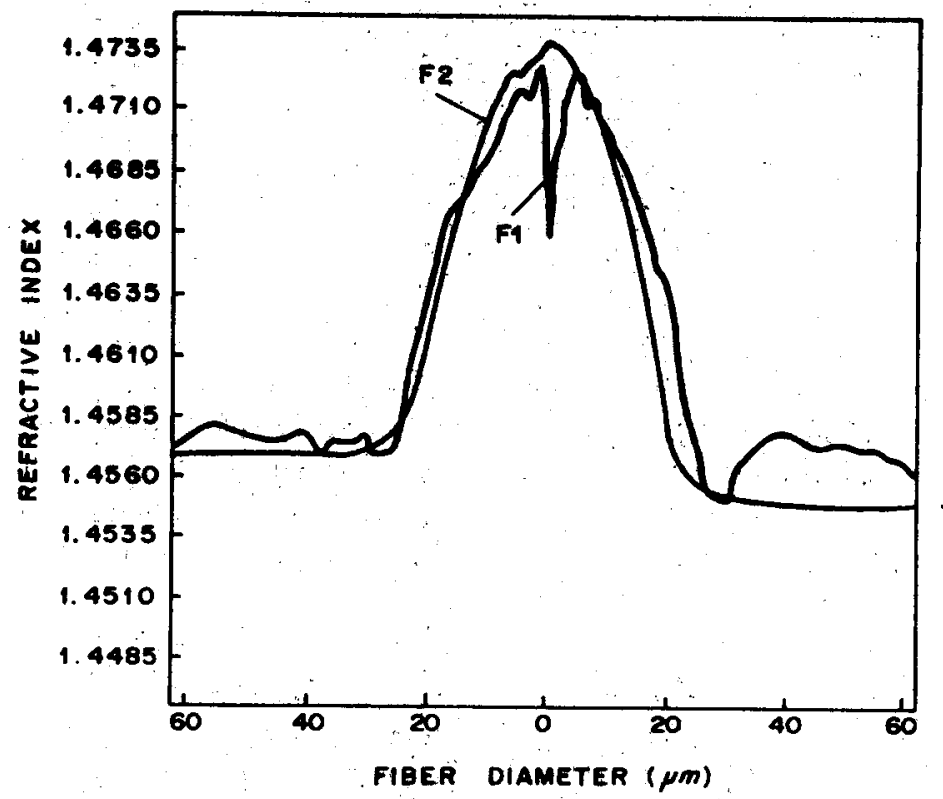

Fig. 2. Measure of refractive index profile of multimode fibers labeled $F 1$ and $F 2$. Note the central dip in the core in $F 1$ :

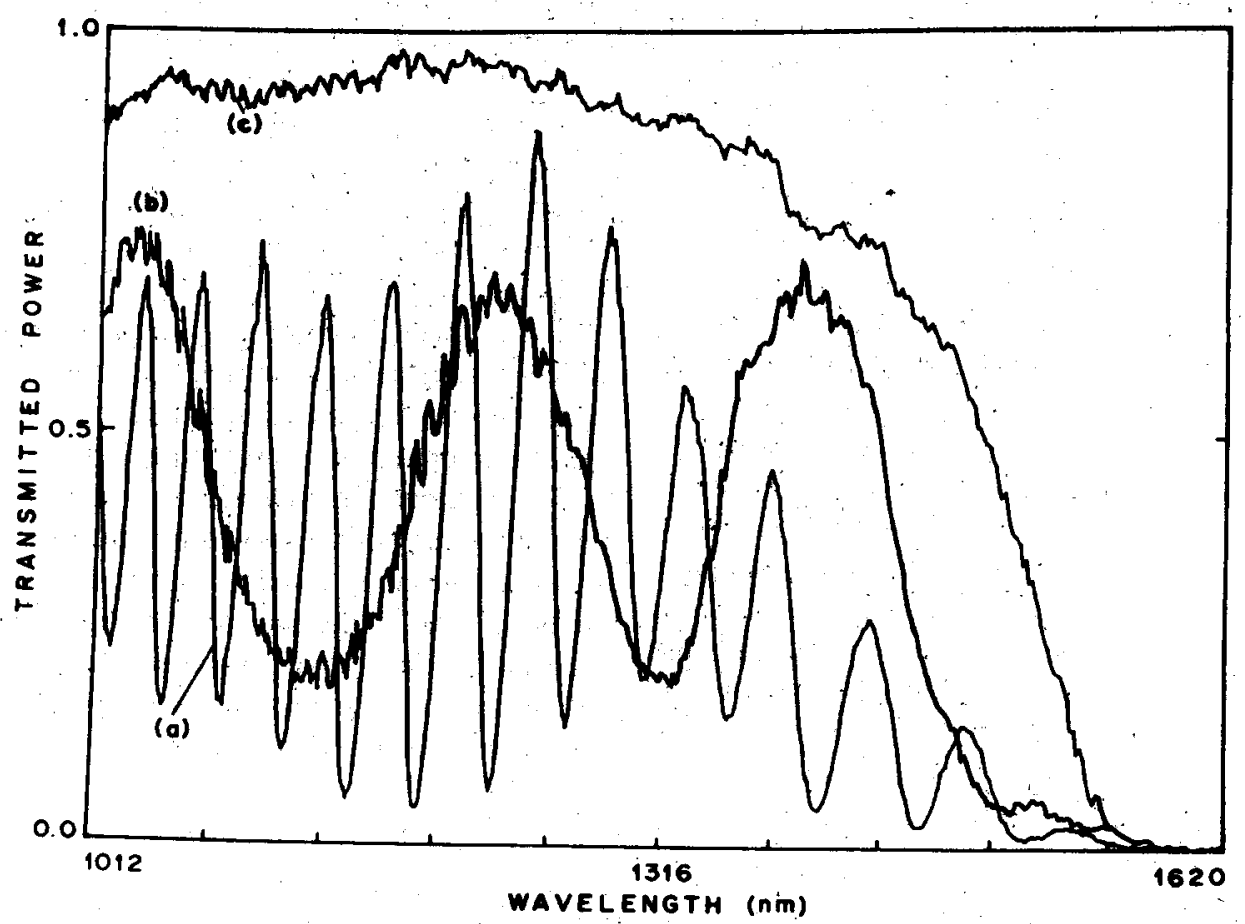

Fig. 3. Transmitted power versus wavelength for untapered multifiber unions using a fiber length $L=12 \mathrm{~cm}$. (a) $F 1$. (b) $F 2$. Line (c) is the overall system response (light source, single-mode fiber, and cladding mode-stripper). 


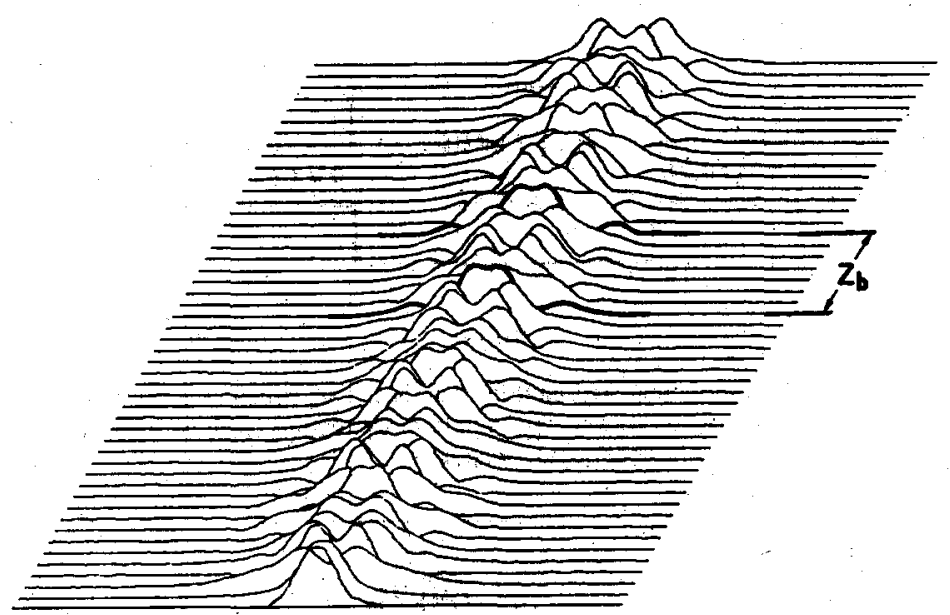

Fig. 4. Generated beating modes for $F 1$. The working wavelength was $\lambda=$ $1300 \mathrm{~nm}$. The simulated length is much smaller than in practice in order to obtain a clearer view of the interference process. $z_{b}$ is the distance for which a repetition of the maxima (or minima) in the power distribution is obtained.

fibers. This similarity gives proof that conically tapered regions are of very small importance to the output power oscillatory behavior. A further point to be indicated is that the output power is independent of the input light polarization. The temperature stability in a range from -5 to $+40^{\circ} \mathrm{C}$ has been observed.

An important point to be considered is the influence of the employed multimode fiber characteristics on the oscillatory behavior in untapered multifiber unions. If lines (a) and (b) in Fig. 3 are compared, some differences can be observed. First, if the same value for $L$ is employed, the peak separation $\Delta \lambda_{p}$ is much smaller with fiber $F 1$ than with $F 2$. And second, oscillation amplitudes are larger for $F 1$ than for $F 2$.

The main difference between $F 1$ and $F 2$ is the small central dip in the refractive index profile for fiber $F 1$. As reported in a recent letter for slab structures [6], this refractive index dip decreases power coupling from input mode to zero-order mode and increases coupling to next-order modes. This effect causes $F 1$ to have an extinction ratio higher than $F 2$.

\section{Theoretical Model}

A qualitative approach for the behavior of our structure can be obtained from the beam propagation method. Fig. 4 shows the obtained power distribution for fiber $F 1$. In this case, $\lambda=$ $1300 \mathrm{~nm}$ was employed as the working wavelength.

Fig. 3 shows a certain modulation. It is due to the existence of more than two modes at our structure. This is the origin of a nonperiodic behavior in the modal field distribution. However, as can be seen in Fig. 4, there is an effective beat length $z_{b}$ being the distance between field intensity maxima, or minima, along $L . z_{b}$ depends both on working wavelength and on fiber characteristics. In this way, BPM indicates that $z_{b}$ is much smaller in fiber $F 1$ than in fiber $F 2\left(z_{b 2} \simeq 4.5 z_{b 1}\right)$, due to the difference between the refractive index profiles.

The peak separation, for any $\lambda$ and $L$, is given by [4]

$$
\Delta \lambda p \simeq \frac{\lambda z_{b}(\lambda)}{L}
$$

$z_{b}$ being the effective beat length. As can be seen in Fig. $4, z_{b 1}$ $\simeq 3.32 \mathrm{~mm}$ for fiber $F 1$.

Fig. 5 shows $\Delta \lambda_{p}$ variation with $L$ for $\lambda=1300 \mathrm{~nm}$. Line (a) gives the results obtained, from (1), for fiber $F 1$. Dots show the experimental values. Results for fiber $F 2$ are given in line (b) (theoretical) and black dots are the experimental values. There is a good agreement between both results.

\section{Conclusion}

As a conclusion, studies of untapered multifiber unions produced from slightly depressed core fibers $(F 1)$ and from usual fibers $(F 2)$ indicate that the power transmission is oscillatory in both cases with the wavelength. This behavior is similar to abruptly tapered single-mode fibers, and has similar potential applications. The advantage of our structure lies in its low cost, as a result of the simple fabrication method.

Due to the oscillatory spectral behavior, untapered multifiber unions are potentially applicable for channel separation in wavelength-multiplexed fiber optic system, by means of an adequate selection of their length and multimode fiber optical characteristics. This would be very useful in a $1.3 / 1.55 \mu \mathrm{m}$ multi/demultiplexer.

It has been shown that refractive-index profile shape influences the peak separation and power oscillation amplitude of the spectral response. Optimization of this profile shape can lead to an optimum transfer function.

The polarization insensitivity, temperature stability, and very low-cost offer a way to practical devices.

Some questions remain unsolved. One is the theoretical study of the influence of misalignment in single-mode/ multimode interfaces. Another one is the study of the difference in excess loss between tapered fibers and untapered multifiber unions. This will be reported elsewhere.

\section{ACKNOWLEDGMENT}

We would like to thank the Laboratory of Electromagnetism and Acustic (Gent, Belgium), Cables de Comunicaciones, Zaragoza, Spain, and F. Arrebola. 


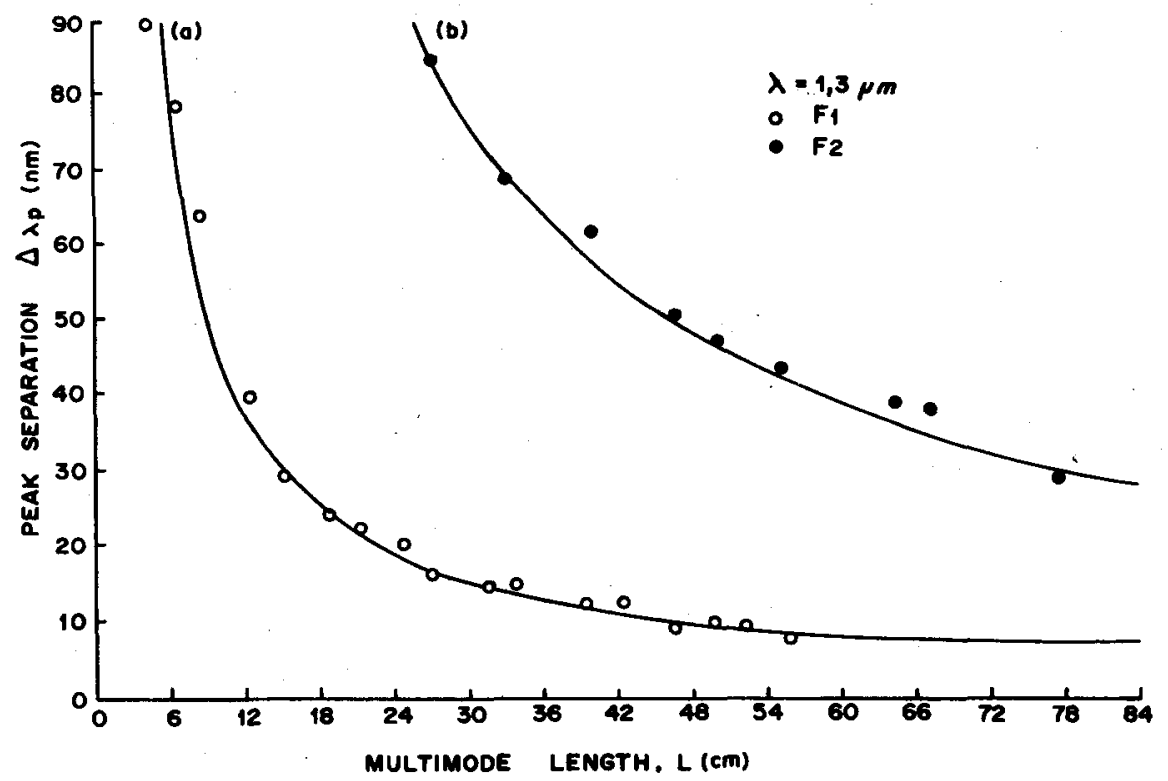

Fig. 5. Peak separation $\left(\Delta \lambda_{p}\right)$ as a function of multimode fiber length $L$ for $\lambda=1300 \mathrm{~nm}$. Line (a) is the theoretical curve (1) and the circles show the experimental points for $F 1$ fiber. Line (b) depicts the theoretical curve for $F 2$ fiber and the black circles are the experimental values.

\section{REFERENCES}

[1] F. Gonthier, J. Lapierre, C. Veilleux, S. Lacroix, and J. Bures, "Investigation of power oscillations along tapered monomode fibers," Appl. Opt., vol. 26, pp. 444-449, 1987.

[2] K. P. Jedrzejewski, F. Martinez, J. D. Minelly, C. D. Hussey, and F. P. Payne, "Tapered-beam expander for single-mode optical-fibre gap devices," Electron. Lett., vol. 22, pp. 105-106, 1986.

[3] S. Lacroix, F. Gonthier, and J. Bures, "All-fiber wavelength filter from successive biconical tapers," Opt. Lett., vol. 11, pp. 671-673, 1986.
[4] P. R. Horche and M. A. Muriel, "Spectral behavior of untapered multi-fiber unions," in Tech. Dig., Conf. Opt. Fiber Commun. (OFC'89), Houston, TX, 1989, paper WQ11.

[5] S. Lacroix, F. Gonthier, R. J. Black, and J. Bures, "Tapered-fiber interferometric wavelength response: The achromatic fringe," Opt. Lett., vol. 13, pp. 395-397, 1988.

[6] M. López-Amo, P. Mendez-Valdés, M. A. Muriel, P. Kaczmarski, and P. E. Lagasse, "Design of two-mode interference wavelength filter utilising symmetric three mode structure," Electron. Lett., vol. 24, pp. 1525-1526, 1988. 